\title{
Les prions et l'hérédité extrachromosomique chez la levure: la rencontre de deux anomalies
}

Un récent article de Wickner (Bethesda, MD, USA) [1], soutenu par un commentaire enthousiaste de Weissmann, propose l'existence d'un phénomène de type prion chez la levure. Quelles caractéristiques communes aux deux systèmes permettent de faire ce rapprochement? Quelles particularités les séparent? Que peut amener le modèle levure dans cet inquiétant problème de santé ?

Les prions ont donné lieu à des nombreuses publications [2, 3]. Je ne retiendrai que quatre de leurs propriétés: (1) le caractère invasif dans la cellule, (2) l'absence apparente d'acide nucléique, (3) l'infectivité et (4) leur résistance aux procédés classiques de stérilisation. Le phénomène décrit par Wickner a une longue histoire cachée. Il y a vingt-cing ans, Lacroute, en étudiant la régulation du métabolisme des pyrimidines chez la levure, décrivait trois mutations urel, ure2 et [URE3]. Les trois phénotypes liés à l'utilisation de l'acide uréidosuccinique se sont avéré être liés à la régulation du métabolisme azoté, par le hasard de similitudes structurales entre cet acide et l'acide allantoïque (voir la revue [4]).

urel est une mutation dans un gène codant pour une glutamate déshydrogénase; ure2 est une mutation dans un gène codant pour un régulateur négatif important du système. Ure2p (la protéine du gène URE2) règle l'activité d'une douzaine de gènes impliqués dans l'utilisation de l'azote [4]. Sa similitude avec les glutathion-S-transférases [5] permet d'ouvrir un champ précis de recherche sur la biochimie du phénomène. [URE3] est le phénomène anormal. Les souches possédant ce déterminant ont exactement les mêmes dérégulations que les mutants ure2. La différence entre les deux est génétique: ure2 se transmet de manière mendélienne. [URE3] est un phénomène invasif. Les diploïdes obtenus par fusion d'un haploïde sauvage avec une souche [URE3] sont [URE3] ('dominance'). Les quatre produits de la méiose sont [URE3]. Enfin, on peut montrer que le déterminant transite par le cytoplasme $[1,6]$. Cela correspond donc au caractère invasif du prion.

Les essais pour cloner le déterminant, ainsi que ceux menés pour identifier un acide nucléique lié à [URE3] ont toujours été vains ([7], F. Lacroute, communication personnelle). D'autres arguments indirects, comme la guérison réversible du phénotype [1] sont également en accord avec l'absence d'acides nucléiques (deuxième propriété des prions)

Le dernier point important est l'exclusion entre les deux types de mutation. Les souches ure2 sont incapables de propager [URE3] [1, 6 . Cela constitue le parallèle de la situation trouvée chez les souris transgéniques délétées pour le gène $\mathrm{Pr}$, qui sont incapables de propager le phénomène $\left(\mathrm{m} / \mathrm{s} n^{\circ} 8-9\right.$, vol. 9 , p. 989) [8].

L'hypothèse de Wickner est donc simple: [URE3] est une forme anormale, non fonctionnelle, destructurée de la protéine codée par le gène chromosomique URE2. Cette forme induit la "destructuration" de la protéine normale, ce qui rend le phénomène invasif et cytoplasmique.

Les deux autres propriétés des prions, les plus inquiétantes, l'infectivité et la résistance à la stérilisation, n'ont jamais pu être mises en évidence, malgré de très nombreux essais [1, 7].

Que peut amener le modèle levure? Contrairement aux prions pour lesquels on ignore tout de la fonction (les souris sans prion n'ont pas de phénotype spécial), le système levure est assez bien connu au niveau physiologique [4]. En particulier, si l'activité glutathion-S-transférase de Ure $2 p$ est confirmée, le rôle du glutathion dans la "destructuration" de Ure2p en [URE3] peut être testé [1]. Les relations entre la fonction régulatrice de Ure2p et la propriété de "destructuration" pourront également être analysées. De manière pertinente, un mutant particulier (ure2-1) produit une protéine Ure2p qui conserve ses propriétés de régulation, mais devient résistante à la "destructuration" en [URE3] [7]. 
L'ensemble de ces données, amplifiées par la facilité de manipulation de l'organisme, permet d'espérer que, avec la compréhension des mécanismes, l'hypothèse du prion comme protéine infectieuse pourrait être définitivement établie.

Pour terminer, il faut s'interroger sur l'aspect historique de cette passionnante résurrection. En 1975, il y a donc bientôt vingt ans, Lacroute et moi-même écrivions : "The possibility of [URE3] entering a cell by a cytoplasmic contribution does not prove that it is a nucleic acid. Another type of molecule (e.g. a protein) can indeed enter the cell "[6]. (Nous pensions alors à une boucle auto-activatrice du gène URE2.)

Wickner a eu l'entêtement de ne pas abandonner ce phénomène insolite, puis la clairvoyance de faire le rapprochement avec les prions. Encore une fois, c'est dans une bizarre anomalie que la réelle nouveauté se cachait, un rappel à méditer dans un temps où les futurs résultats tiennent souvent lieu de projets de recherche...

M.A.

1. W'ickner RB. [URE3] as an altered URE2 protein : evidence for a prion analog in Saccharomyces cererisiae. Science 1994 ; 264 : 566-9. 2. Pagès C. Encéphalopathies spongiformes et prions, liaison fatale. médecine/sciences 1993 ; 9 : 1404-8.

3. Bussard A. La découverte des prions vat-elle révolutionner la biologie moléculaire? médecine/sciences 1993; 9: 1409-10.

4. Magasanik B. Regulation of nitrogen utilization. In: The molecular and cellular biology of the yeast Saccharomyces. Cold Spring Harbor Laboratory Press 1992: 283 -318.

5. Coshigano PW, Miller SM, Magasanik B. Physiological and genetic analysis of the carbon regulation of the NAD-dependent GDM of S. cerevisiae. Mol Cell Biol 1991; 11 : 44-55. 6. Aigle M, Lacroute F. Genetical aspects of [URE3], a nonmitochondrial, cytoplasmically inherited mutation in yeast. Mol Gen Genet $1975 ; 136: 327-35$

7. Aigle M. Contribution à l'étude de l'hérédité non-chromosomique de Saccharømyces cerevisiae: facteur [URE 3] et plasmides hybrides. Thèse ì l'Université L.-Pasteur, Strasbourg. 1979. 8. Büeler H, Aguzzi A, Sailer A, Greiner RA, Autenried P, Aguet M, Weissman C. Mice devoid of PrP are resistant to Scrapie. Cell $1993 ; 73: 1339-47$

$\mathrm{m} / \mathrm{s} n^{\circ}$ 6-7 vol. 1(), juin-juillet 94 contrôle négativement l'expression du gène lin-14 semble être la synthèse d'ARN anti-sens s'hybridant à l'extrémité 3' non codante du messager lin-14 et en bloquant la traduction par un mécanisme encore non précisé $[2,3]$. Ce mécanisme est ainsi l'un des exemples du rôle des régions 3' non traduites des ARN messagers qui apparaît jour après jour plus important, notamment au cours du développement $\left(\mathrm{m} / \mathrm{s} n^{\circ} 4\right.$, vol. 10 , p. 464).

[1. Labouesse M. médecine/sciences $1994 ; 10$ : 337-41.]

[2. Lee RC, et al. Cell 1993; 75 : 843-54.]

[3. Wightman I, et al. Cell 1993; 75 : 855-62.]

in-14 lf (pour lin-14 loss-of-function) ont un développement larvaire précoce, les événements caractéristiques des stades ultérieurs débutant immédiatement après l'éclosion de l'embryon. En revanche, les mutants avec gain de fonction (lingf pour lin-14 gain-of-function) ont un premier stade larvaire normal, mais un retard et une absence de l'évolution ultérieure qui est remplacée par une réitération des événements du premier stade. Les mutations du gène lin-4 ont des conséquences inverses : développement retardé pour les mutants lin-4 lf et accéléré pour les mutants lin-4 gf. lin-14 code pour une protéine LIN-14 de localisation nucléaire. Certains mutants avec gain de fonction sont caractérisés par une délétion de l'extrémité 3' non codante du messager lin-14 qui comporte plusieurs éléments répétés. En revanche, lin-4 semble aboutir à la synthèse de petits ARN non codants dont l'un est complémentaire des éléments répétés de l'extrémité 3' non codante du messager lin-14. Au premier stade larvaire, la protéine LIN-14 est abondante, et les transcrits lin-4 sont absents. En revanche, les transcrits lin-4 apparaissent aux stades ultérieurs, parallèlement à une diminution et à une disparition de la protéine LIN-14. Par conséquent, le mécanisme par lequel le gène lin-4
Un facteur de régénération des îlots de Langerhans. Une équipe japonaise dirigée par Okamoto a, en 1988, décrit l'ADNc codant pour une protéine de 165 acides aminés synthétisée dans des îlots de Langerhans en régénération [1]. Watanabe et al., de la même équipe, démontrent maintenant que l'administration de la protéine recombinante correspondante, dénommée Reg, à des rats ayant subi une pancréatectomie de $90 \%$, favorisait la régénération des cellules $\beta$ langerhansiennes [2]. Reg augmente l'incorporation de cellules $\beta$, favorise la croissance cellulaire, diminue l'hyperglycémie postpancréatectomie et améliore l'état pondéral des animaux opérés. La protéine Reg semble donc être un facteur de croisssance des cellules $\beta$ des îlots de Langerhans et pourrait constituer un traitement d'avenir du diabète humain en facilitant la régénération des cellules $\beta$ soumises à une agression auto-immune.

[1. Terazono K, et al. I Biol Chem 1988; 263: 2111-4.]

[2. Watanabe, et al. Proc Natl Acad Sci USA 1994; 91 : 3589-92.] $[3 \mathrm{H}]$ thymidine dans le noyau des 